\title{
Oncology-Inspired Treatment Options for COVID-19
}

\author{
Nagavarakishore Pillarsetty ${ }^{1}$, Lukas M. Carter ${ }^{1}$, Jason S. Lewis ${ }^{1,2}$, and Thomas Reiner ${ }^{1,3}$ \\ ${ }^{1}$ Department of Radiology, Memorial Sloan Kettering Cancer Center, New York, New York; ${ }^{2}$ Molecular Pharmacology Program, \\ Memorial Sloan Kettering Cancer Center, New York, New York; and ${ }^{3}$ Chemical Biology Program, Memorial Sloan Kettering Cancer \\ Center, New York, New York
}

\begin{abstract}
CR3022 is a human antibody that binds to severe acute respiratory syndrome coronavirus 2 (SARS-CoV-2). Here, we explore the use of CR3022 as a molecularly targeted radiotherapeutic. Methods: CR3022 was labeled with ${ }^{131} \mid$ and purified, yielding ${ }^{131} \mid-C R 3022$. Using a magnetic bead assay and a recombinant SARS-CoV-2 spike protein fragment, we tested binding of ${ }^{131}$ I-CR3022 in the presence and absence of CR3022. Results: We conjugated the antibody CR3022 with a purity of more than $98 \%$ and a specific activity of more than $292 \mathrm{MBq} / \mathrm{mg}$. Using a bead-based assay, we confirmed that binding of ${ }^{131} \mathrm{I}-\mathrm{CR} 3022$ is selective and is significantly reduced in the presence of unlabeled antibody $(3.14 \% \pm$ $0.14 \%$ specific uptake and $0.10 \% \pm 0.01 \%$ specific uptake, respectively; $P<0.0001)$. Conclusion: Our results confirm the potential of CR3022 as a molecularly targeted probe for SARS-CoV-2. A labeled version of CR3022 could potentially be used for Auger radiotherapy or noninvasive imaging.
\end{abstract}

Key Words: auger; radiotherapy; COVID-19; SARS-CoV-2; CR3022

J Nucl Med 2020; 61:1720-1723

DOI: $10.2967 /$ jnumed.120.249748

\section{$\mathbf{R}$}

adiotherapy, the treatment of disease with ionizing radiation, plays a significant role in the treatment and management of cancer. Most recently, molecularly targeted endoradiotherapeutics have received significant attention (1). These agents consist of a targeted vector (a small molecule, a peptide, or an antibody) and a radioactive payload (an $\alpha$-emitter, a $\beta$-emitter, or an Auger emitter). Some of these treatments have generated impressive responses, such as was seen for ${ }^{177} \mathrm{Lu}$-DOTATATE (Lutathera; Advanced Accelerator Applications), a $\beta$-emitter recently approved by the Food and Drug Administration that has a half-life of $6.7 \mathrm{~d}$ and extends both progression-free and overall survival in the setting of midgut neuroendocrine tumors (2).

For molecularly targeted endoradiotherapeutics, particular focus has to be placed on the kind of radioactive emitter, as different disintegration pathways produce particle emissions of varying type and profile $(3,4)$. Matching the half-life and decay type to a

Received May 14, 2020; revision accepted Jun. 23, 2020.

For correspondence or reprints contact: Thomas Reiner, Memorial Sloan Kettering Cancer Center, 1275 York Ave., New York, NY 10065.

E-mail: reinert@mskcc.org

Published online Jul. 17, 2020.

Immediate Open Access: Creative Commons Attribution 4.0 International License (CC BY) allows users to share and adapt with attribution, excluding materials credited to previous publications. License: https://creativecommons. org/licenses/by/4.0/. Details: http://jnm.snmjournals.org/site/misc/permission. xhtml.

COPYRIGHT @ 2020 by the Society of Nuclear Medicine and Molecular Imaging. particular application is therefore imperative and can determine success or failure.

The most recognized radioactive emissions of therapeutic relevance are $\alpha$ - or $\beta$-particles, which represent a ${ }_{2}^{4} \mathrm{He}^{2+}$ nucleus and an electron, respectively. $\alpha$-emitting radioisotopes have particle pathlengths of 50-100 $\mu \mathrm{m}$ and high linear energy transfer (5) rates $(80 \mathrm{keV} / \mu \mathrm{m}) . \beta$-emitting radioisotopes have particle pathlengths of up to several millimeters in soft tissue and significantly lower linear energy transfer rates $(\sim 0.2 \mathrm{keV} / \mu \mathrm{m})$. With the coronavirus disease 2019 (COVID-19) pandemic in mind, both $\alpha$ - and $\beta$-particles are therefore likely suboptimal therapeutics, considering severe acute respiratory syndrome coronavirus 2 (SARS-CoV-2) virions' diameters (80-120 $\mathrm{nM}(6))$ and the associated potential for detrimental effects on surrounding tissue. However, Auger electron emitters appear to be of particular significance here. Auger electrons combine a relatively high linear energy transfer $(4-26 \mathrm{keV} / \mu \mathrm{m})$ with extremely short nanometer-to-micrometer particle pathlengths, concentrating their cytotoxic potential into minute volumes compared with cellular dimensions. In a rough approximation, Auger electrons with energies of between 0.5 and $10 \mathrm{keV}$ are sufficiently energetic to penetrate deep into the virus, producing direct and indirect radiologic effects (i.e., therapeutic action) when originating at the viral envelope, but are insufficiently energetic to directly damage neighboring cell nuclei.

In oncology, such characteristics led to attempts to incorporate Auger electrons into artificial nucleotides in order to treat cancers by causing complex DNA damage in tumor cells, but this approach has ultimately not been successful because of the difficulty of delivering lethal doses to a large enough fraction of tumor cells within a particular lesion (7-9). Recent preclinical work using different delivery mechanisms suggests a renewed promise and corroborates the considerable toxic effect of Auger emitters on tumor cells (10). In Figure 1, we illustrate the relationship between Auger/conversion electron energy and yield for ${ }^{125}$ I. Examples for other prolific Auger emitters (11) can be found in Figure 2.

${ }^{125} \mathrm{I}$ is reactor-produced and available in large quantities. At the time of writing, the McMaster nuclear reactor in Hamilton, Ontario, produces the isotope predominantly for brachytherapy, allowing treatment of 70,000 patients annually.

In the past, ${ }^{125}$ I was explored as an Auger-based radiotherapeutic for a genetically engineered measles virus. The virus, which expressed the sodium iodide symporter in infected cells, was sensitive to ${ }^{125} \mathrm{I}$ in vitro, where virus replication could be stopped. These results, however, did not translate to an in vivo model, suggesting suboptimal pharmacokinetics of ${ }^{125}$ I-iodide (12). A selective, molecularly targeted vector such as the monoclonal antibody CR3022 could serve as a delivery agent for ${ }^{125}$ I. CR3022 binds to the SARS-CoV-2 receptor binding domain with a dissociation 


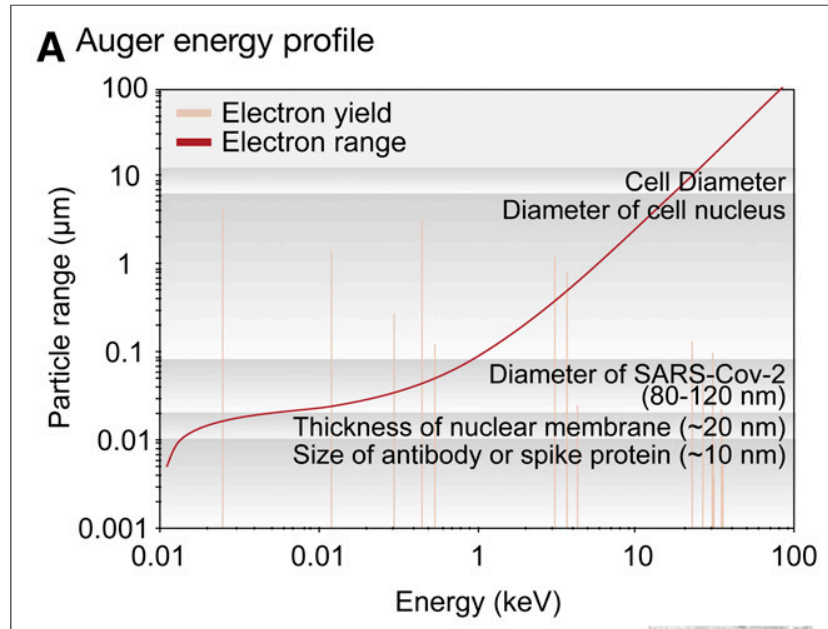

B SARS-CoV-2 Radiotherapy

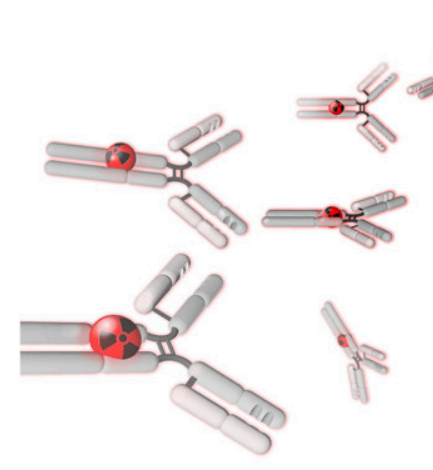

C

Tracer radiosynthesis
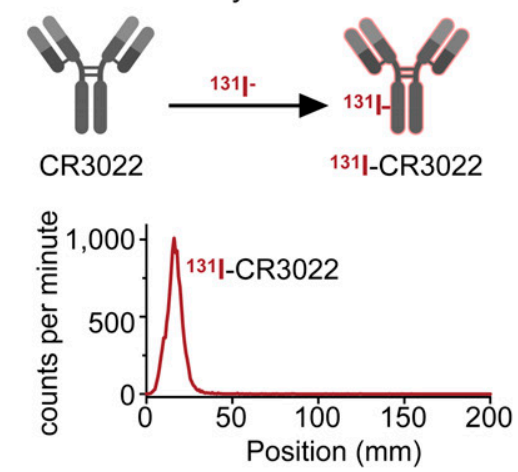

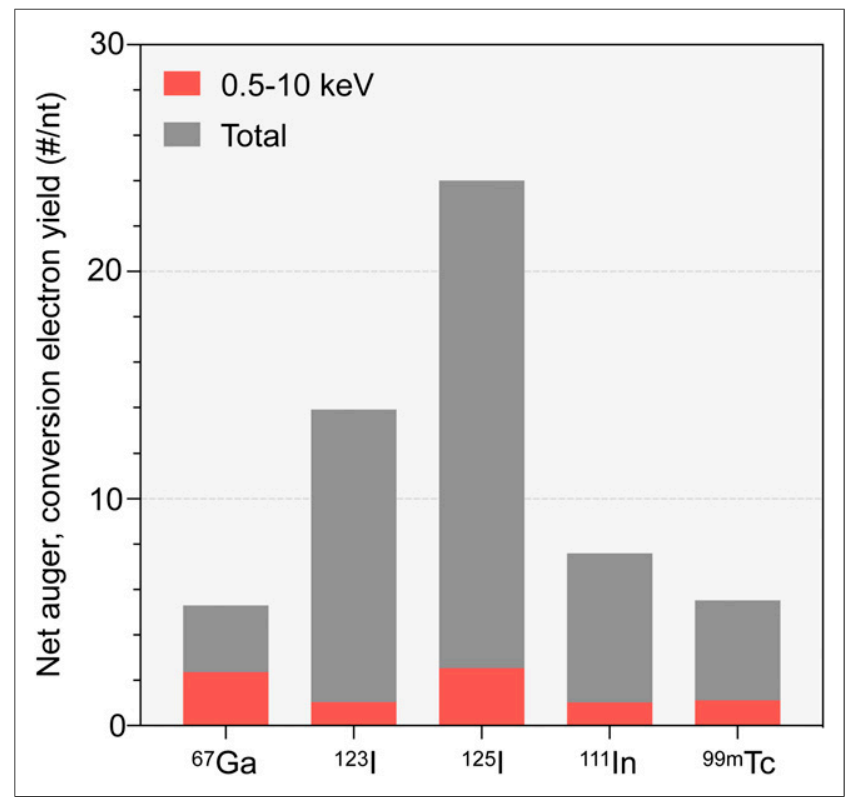

FIGURE 2. Net yields of monoenergetic electrons (Auger, conversion electrons) per nuclear transformation for ${ }^{67} \mathrm{Ga},{ }^{123} \mathrm{I},{ }^{125} \mathrm{I},{ }^{111} \mathrm{In}$, and ${ }^{99 \mathrm{~m} T \mathrm{C} .}$ Red bars represent contribution, to total yield, of electrons within 0.5 - to 10-keV energy range. Yields were obtained from ICRP publication 107 (29).

Another iodine isotope, ${ }^{131} \mathrm{I}$, not only is used as a standard-ofcare treatment for certain types of thyroid cancers but also finds widespread use in scintigraphy and whole-body SPECT imaging. Intuitively, a radiolabeled CR3022 could be valuable for imaging, potentially serving as a direct, spatially resolved, contemporaneous, and noninvasive readout of viral load within a patient. From a drug-development perspective, a direct readout of SARS-CoV-2 viral load could represent a quick, upstream indicator of therapy success. This tool could be particularly interesting for clinical research, and similar approaches have been used to accelerate oncologic drug development pipelines in the past (15).

\section{MATERIALS AND METHODS}

\section{General}

All reagents were obtained from commercial vendors and used without further purification. Phosphate-buffered saline (PBS), 0.9\%; Iodo-Gen (1,3,4,6-tetrachloro-3 $\alpha, 6 \alpha$-diphenyl-glycoluril; Pierce Biotechnology, Inc.); and dichloromethane were obtained from Thermo Fisher Scientific. Anti-SARS-CoV-2 antibody CR3022 was purchased from Creative Biolabs. Recombinant SARS-CoV-2 spike protein, subunit S1 (host cell receptor binding domain-receptor binding domain), with an N-terminal histidine tag was purchased from Raybiotech. Magnetic beads $1 \mu \mathrm{m}$ in diameter and functionalized with HisPur nickel-nitrilotriacetic acid for the bead assay were purchased from Thermo Fisher Scientific. Iodo-Gen-coated glass reaction tubes were prepared by evaporating $50 \mu \mathrm{L}$ of Iodo-Gen solution $(50 \mu \mathrm{g}, 1 \mathrm{mg} /$ $\mathrm{mL})$ in a borosilicate glass test tube $(12 \times 75 \mathrm{~mm})$. PD MiniTrap G-25 columns (GE Healthcare) were preconditioned with $2 \mathrm{~mL}$ of PBS before being used to separate radioiodinated antibody from the free radioiodine.

\section{Radiosynthesis}

A $70-\mu \mathrm{L}$ volume of PBS was added to an Iodo-Gen $(100 \mu \mathrm{g})-$ precoated culture tube. To the resulting solution, a $25-\mu \mathrm{g}$ quantity SARS-CoV-2 but also, potentially, related diseases $(13,14)$. 
of CR3022 monoclonal antibody $(25 \mu \mathrm{L}, 1.0 \mathrm{mg} / \mathrm{mL})$ was added. A 9.25-MBq $(250 \mu \mathrm{Ci})$ quantity of ${ }^{131} \mathrm{I}-\mathrm{NaI}$ (in $17 \mu \mathrm{L}$ of $0.1 \mathrm{~N} \mathrm{NaOH}$ ) was added to the solution in the tube, and the mixture was allowed to react for $4 \mathrm{~min}$ at room temperature before being loaded onto a PD MiniTrap G-25 column (GE Healthcare) preconditioned with $2 \mathrm{~mL}$ of PBS. The radiolabeled antibody was purified using saline as the eluant. Fraction 3 was used for the binding studies. The purity of the compound was measured using silica gel instant thin-layer chromatography paper, with $10 \%$ trifuloroacetic acid in water as the eluent. The specific activity of the final product was $292 \mathrm{MBq} / \mathrm{mg}(7.9 \mathrm{mCi} / \mathrm{mg}$; $177.5 \mu \mathrm{Ci} / 22.5 \mu \mathrm{g})$.

\section{Magnetic Bead Assay}

We have previously described the details of the bead-based assay (16). The assay comprises 3 separate arms - a control with no antigen, a positive control, and a blocking control—and each arm analyzes samples in triplicate. The first arm serves as a control to measure nonspecific binding of the radioligand to the beads without any target antigen (SARS-CoV-2 spike protein, subunit S1), the second arm assesses radioligand binding to beads coated with this antigen, and the third arm validates the specificity of radioligand binding to the cognate antigen in the presence of an excess of unlabeled ligand.

Briefly, samples were prepared by aliquoting $20 \mu \mathrm{L}$ of the magnetic bead slurry into a 1.5-mL LoBind microcentrifuge tube (Fisher Scientific). The beads were washed by adding $380 \mu \mathrm{L}$ of PBS containing $1 \%$ bovine serum albumin. The tubes were placed in a vortex mixer for $5 \mathrm{~s}$, followed by a brief spin in a mini-centrifuge before being placed on a magnetic rack (DynaMag-2; ThermoFisher Scientific) for $30-45 \mathrm{~s}$ to isolate the magnetic beads. The SARS-CoV-2, subunit $\mathrm{S} 1$, antigen was resuspended to achieve a concentration of a $0.1 \mathrm{mg} /$ $\mathrm{mL}$. The washed beads were resuspended in $390 \mu \mathrm{L}$ of PBS-bovine serum albumin, and the beads in all tubes except the control arm were incubated with $1 \mu \mathrm{g}(10 \mu \mathrm{L})$ of His-tagged or biotinylated antigen for $15 \mathrm{~min}$ on an Eppendorf Thermomixer at $300 \mathrm{rpm}$ at room temperature. Subsequently, the beads were washed once with $400 \mu \mathrm{L}$ of PBS-T before the addition of $1 \mathrm{ng}$ of the radiolabeled antibody ( $\left.{ }^{131} \mathrm{I}-\mathrm{CR} 3022\right)$ and resuspension in $1 \%$ bovine serum albumin-PBS. ${ }^{131}$ I-CR3022 was incubated with antigen-coated beads for $30 \mathrm{~min}$ on a rotating mixer at room temperature. A large excess $(5 \mu \mathrm{g})$ of the unlabeled cold antibody CR3022 was added a few seconds before $1 \mathrm{ng}$ of the radioligand was added to antigen-coated beads in the blocking arm. Thereafter, the beads were isolated using a magnet, and the supernatant containing unbound radioligand was aspirated with a pipette and collected in separate tubes. To remove nonspecifically bound radioligand, the beads were washed twice with $400 \mu \mathrm{L}$ of PBS-bovine serum albumin. Finally, the beads, supernatant, and washes were measured for radioactivity on a $\gamma$-counter. The relative binding fractions were determined by dividing the percentage of total activity bound to magnetic beads to the total activity (beads + supernatants + wash).

\section{RESULTS AND DISCUSSION}

SARS-CoV-2 is a coronavirus that emerged in late 2019 and has resulted in an ongoing pandemic, causing cases of COVID-19 across the globe. Common symptoms include fever, cough, shortness of breath, and muscle aches (17). SARS-CoV-2 enters host cells via the angiotensin-converting enzyme 2 receptor, which is expressed in type II alveolar cells of the lungs, and can severely affect lung function $(18,19)$.

Intuitively, many lessons learned from attempts to treat tumors with Auger emitters could be adapted for radiotherapeutically inactivating extracellularly circulating SARS-CoV-2 in patients. After all, tumor cells and SARS-CoV-2 share an important hallmark in their ability to evade patients' immune systems $(20,21)$. However, whereas tumor cells may permanently escape the immune system (or only become tolerant over the course of the disease), pathogens such as SARS-CoV-2 can be efficiently eliminated once adaptive immunity has been acquired (22). Although it is unlikely that treatment of SARS-CoV-2, mediated by a radiotherapeutic Auger emitter, can eliminate all virions, radiotherapy could be used in combination with other treatments and consequently improve outcomes (Fig. 1). Such treatment combinations could include currently tested treatments such as anti-IL-6 antibodies or remdesivir. However, radiation therapy was reported to initiate and influence the inflammatory and immune system (23), and care has to be taken that there is no subsequent increase in the likelihood of cytokine storms (24).

As a proof of the concept that molecular targeting of SARS$\mathrm{CoV}-2$ is possible, we turned to CR3022, a human IgG1 antibody constructed from RNA, which was isolated from the lymphocytes of a convalescent SARS-CoV patient from Singapore (25). Although CR3022 is therefore a potent binder of the SARS-CoV-2 receptor binding domain, the recognized epitope does not overlap the angiotensin-converting enzyme 2 binding site (the receptor binding motif), and CR3022 consequently does not compete with angiotensin-converting enzyme 2 for binding to SARS-CoV-2. This characteristic is notably not a drawback for Auger radiotherapy of SARS-COV-2. Using Iodo-Gen for iodination, a method established both in preclinical and clinical settings $(26,27)$, we covalently conjugated ${ }^{131}$ I to commercially available CR3022 with a purity of more than $98 \%$ and a specific activity of $292 \mathrm{MBq} / \mathrm{mg}$ (Fig. 1C). We confirmed that the modified ${ }^{131}$ I-CR3022 retained its potent binding to SARS-CoV-2 using a magnetic bead assay, testing its binding to a recombinant His-tagged SARS-CoV-2 receptor binding domain.

The specific binding of ${ }^{131}$ I-CR3022 alone was significantly higher without preincubation of unlabeled CR3022, confirming both that CR3022 binds to SARS-CoV-2 and that binding is not perturbed after covalent modification with ${ }^{131} \mathrm{I}$ (3.14\% $\pm 0.14 \%$ and $0.10 \% \pm 0.01 \%$ specific uptake for ${ }^{131} \mathrm{I}-\mathrm{CR} 3022$ and CR3022, respectively; $P<0.0001$; Fig. 1D). We consider this experiment a potent first step toward translating an orthogonal therapeutic approach for SARS-CoV-2, which could potentially be used as a combination therapy or monotherapy for patients with active infection. The translational hurdles for such a drug could be lower than with traditional therapeutics or vaccines because the pharmacokinetics (which are dictated by the antibody) are decoupled from the pharmacodynamics (dictated by the radioisotope). Although both work synergistically, they can be optimized separately, similar to what has been done for ${ }^{177}$ Lu-PSMA and ${ }^{225}$ Ac-PSMA, two anticancer radiotherapeutics (28). Substitution of the isotope preserved the pharmacokinetic profile while simultaneously showing therapeutic efficacy in patients with acquired resistance to ${ }^{177}$ Lu-PSMA.

Lastly, the integration of other, previously oncologically deployed, strategies could lead to the rapid rollout of SARS-CoV-2 therapeutics as well, including the conjugation of drug conjugates for treating affected cells, or known antigens for efficiently decloaking SARS$\mathrm{CoV}-2$ from the immune system.

\section{CONCLUSION}

Our preliminary data, in combination with the available literature, suggest further development of a radiotherapeutic CR3022, which would be merging different pharmacologic approaches. 


\section{DISCLOSURE}

This work was supported by National Institutes of Health grants R01 CA204441, R35 CA232130, and P30 CA008748. No other potential conflict of interest relevant to this article was reported.

\section{ACKNOWLEDGMENTS}

We thank the Small Animal Imaging Core, the Radiochemistry and Molecular Imaging Probes Core, and the Molecular Cytology Core at Memorial Sloan Kettering Cancer Center. The also thank the Tow Foundation and Memorial Sloan Kettering Cancer Center's Center for Molecular Imaging and Nanotechnology (CMINT) and the Memorial Sloan Kettering Cancer Center Imaging and Radiation Sciences Program.

\section{KEY POINTS}

QUESTION: Can the human monoclonal antibody CR3022 be used as a specific targeted vector for shuttling activity to SARS-CoV-2 virions?

PERTINENT FINDINGS: Labeling of CR3022 is possible, and binding affinity of the antibody for the SARS-CoV-2 receptor binding domain is retained.

IMPLICATIONS FOR PATIENT CARE: CR3022, modified with a radiolabel, could be used for direct imaging of SARS-CoV-2 but also potentially as an Auger radiotherapeutic in patients with active infection.

\section{REFERENCES}

1. Jadvar H. Targeted radionuclide therapy: an evolution toward precision cancer treatment. AJR. 2017;209:277-288.

2. Strosberg J, El-Haddad G, Wolin E, et al. Phase 3 trial of ${ }^{177}$ Lu-dotatate for midgut neuroendocrine tumors. N Engl J Med. 2017;376:125-135.

3. Poty S, Francesconi LC, McDevitt MR, Morris MJ, Lewis JS. $\alpha$-emitters for radiotherapy: from basic radiochemistry to clinical studies-part 2. J Nucl Med. 2018;59:1020-1027.

4. Blower PJ. A nuclear chocolate box: the periodic table of nuclear medicine. Dalton Trans. 2015;44:4819-4844.

5. O'Connor JP, Aboagye EO, Adams JE, et al. Imaging biomarker roadmap for cancer studies. Nat Rev Clin Oncol. 2017;14:169-186.

6. Liu C, Yang Y, Gao Y, et al. Viral architecture of SARS-CoV-2 with post-fusion spike revealed by cryo-EM. bioRxiv website. https://www.biorxiv.org/content/ 10.1101/2020.03.02.972927v1. Published March 5, 2020. Accessed October 7, 2020.

7. Hofer KG, Hughes WL. Radiotoxicity of intranuclear tritium, 125 iodine and 131 iodine. Radiat Res. 1971;47:94-101.

8. Kassis AI. Cancer therapy with Auger electrons: are we almost there? J Nucl Med. 2003;44:1479-1481.

9. Bavelaar BM, Lee BQ, Gill MR, Falzone N, Vallis KA. Subcellular targeting of theranostic radionuclides. Front Pharmacol. 2018;9:996.
10. Pirovano G, Jannetti SA, Carter LM, et al. Targeted brain tumor radiotherapy using an Auger emitter. Clin Cancer Res. 2020;26:2871-2881.

11. Ku A, Facca VJ, Cai Z, Reilly RM. Auger electrons for cancer therapy: a review. EJNMMI Radiopharm Chem. 2019;4:27-36.

12. Dingli D, Peng K-W, Harvey ME, et al. Interaction of measles virus vectors with Auger electron emitting radioisotopes. Biochem Biophys Res Commun. 2005;337: 22-29.

13. Tian X, Li C, Huang A, et al. Potent binding of 2019 novel coronavirus spike protein by a SARS coronavirus-specific human monoclonal antibody. Emerg Microbes Infect. 2020;9:382-385.

14. Yuan M, Wu NC, Zhu X, et al. A highly conserved cryptic epitope in the receptor binding domains of SARS-CoV-2 and SARS-CoV. Science. 2020;368: 630-633.

15. Wang Y, Ayres KL, Goldman DA, et al. ${ }^{18} \mathrm{~F}$-fluoroestradiol PET/CT measurement of estrogen receptor suppression during a phase I trial of the novel estrogen receptor-targeted therapeutic GDC-0810: using an imaging biomarker to guide drug dosage in subsequent trials. Clin Cancer Res. 2017;23:3053-3060.

16. Sharma SK, Lyashchenko SK, Park HA, et al. A rapid bead-based radioligand binding assay for the determination of target-binding fraction and quality control of radiopharmaceuticals. Nucl Med Biol. 2019;71:32-38.

17. Chen N, Zhou M, Dong X, et al. Epidemiological and clinical characteristics of 99 cases of 2019 novel coronavirus pneumonia in Wuhan, China: a descriptive study. Lancet. 2020;395:507-513.

18. Hoffmann M, Kleine-Weber H, Schroeder S, et al. SARS-CoV-2 cell entry depends on ACE2 and TMPRSS2 and is blocked by a clinically proven protease inhibitor. Cell. 2020;181:271-280.e8.

19. Walls AC, Park Y-J, Tortorici MA, Wall A, McGuire AT, Veesler D. Structure, function, and antigenicity of the SARS-CoV-2 spike glycoprotein. Cell. 2020;181:281292.e6.

20. Willimsky G, Blankenstein T. Sporadic immunogenic tumours avoid destruction by inducing T-cell tolerance. Nature. 2005;437:141-146.

21. Vesely MD, Kershaw MH, Schreiber RD, Smyth MJ. Natural innate and adaptive immunity to cancer. Annu Rev Immunol. 2011;29:235-271.

22. Pancer Z, Cooper MD. The evolution of adaptive immunity. Annu Rev Immunol. 2006;24:497-518.

23. McKelvey KJ, Hudson AL, Back M, Eade T, Diakos CI. Radiation, inflammation and the immune response in cancer. Mamm Genome. 2018;29:843865.

24. Ye Q, Wang B, Mao J. The pathogenesis and treatment of the 'cytokine storm' in COVID-19. J Infect. 2020;80:607-613.

25. ter Meulen J, Van Den Brink EN, Poon LL, et al. Human monoclonal antibody combination against SARS coronavirus: synergy and coverage of escape mutants. PLoS Med. 2006;3:e237.

26. Cheung N-KV, Landmeier B, Neely J, et al. Complete tumor ablation with iodine 131-radiolabeled disialoganglioside GD2-specific monoclonal antibody against human neuroblastoma xenografted in nude mice. J Natl Cancer Inst. 1986;77:739_ 745 .

27. Kramer K, Humm JL, Souweidane MM, et al. Phase I study of targeted radioimmunotherapy for leptomeningeal cancers using intra-Ommaya 131-I-3F8. J Clin Oncol. 2007;25:5465-5470.

28. Kratochwil C, Bruchertseifer F, Giesel FL, et al. ${ }^{225}$ Ac-PSMA-617 for PSMAtargeted $\alpha$-radiation therapy of metastatic castration-resistant prostate cancer. J Nucl Med. 2016;57:1941-1944.

29. Eckerman K, Endo A. ICRP publication 107: nuclear decay data for dosimetric calculations. Ann ICRP. 2008;38:7-96. 